\title{
Temperature-FORC analysis of a magnetocaloric Heusler alloy using a unified driving force approach (T*FORC)
}

Cite as: J. Appl. Phys. 127, 133902 (2020); https://doi.org/10.1063/5.0005076

Submitted: 17 February 2020 . Accepted: 24 March 2020. Published Online: 02 April 2020

Victorino Franco (D)

\section{COLLECTIONS}

Paper published as part of the special topic on Multicalorics Note: This paper is part of the Special Topic on Multicalorics.

EP This paper was selected as an Editor's Pick
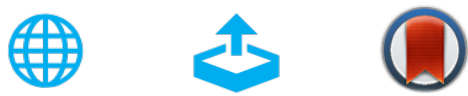

\section{ARTICLES YOU MAY BE INTERESTED IN}

Atomic simulation of interaction mechanism between dislocation and amorphous phase in dual-phase crystalline/amorphous Mg/MgAl alloys

Journal of Applied Physics 127, 135105 (2020); https://doi.org/10.1063/1.5143721

Room temperature multiferroic properties of electrospun gallium ferrite nanofibers Journal of Applied Physics 127, 134101 (2020); https://doi.org/10.1063/1.5142912

Dissipative couplings in cavity magnonics

Journal of Applied Physics 127, 130901 (2020); https://doi.org/10.1063/1.5144202

\section{Lock-in Amplifiers up to $600 \mathrm{MHz}$}
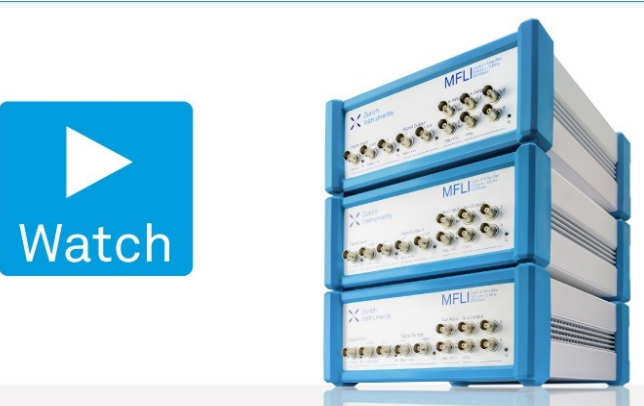


\title{
Temperature-FORC analysis of a magnetocaloric Heusler alloy using a unified driving force approach (T*FORC)
}

Cite as: J. Appl. Phys. 127, 133902 (2020); doi: $10.1063 / 5.0005076$

Submitted: 17 February 2020 - Accepted: 24 March 2020 .

Published Online: 2 April 2020

Victorino Franco ${ }^{\text {a) }}$ (iD

\author{
AFFILIATIONS \\ Dpto. Física de la Materia Condensada, ICMS-CSIC, Universidad de Sevilla, P.O. Box 1065, 41080 Sevilla, Spain
}

Note: This paper is part of the Special Topic on Multicalorics.

${ }^{a}$ Author to whom correspondence should be addressed: vfranco@us.es

\begin{abstract}
Temperature-first order reversal curve (FORC) distributions of thermomagnetic phase transitions are a fingerprinting tool to identify features of the phase transformations of the material. However, they have two major limitations: qualitative character, due to the shift of the loops with increasing driving forces, and long experimental time. The use of an effective temperature that takes into account the different driving forces affecting the transformation allows for a more quantitative comparison of the features of the FORC distributions, as it eliminates the need for an $a d$ hoc selection of the origin of the distribution axes. At the same time, experimental measurements as a function of this effective temperature are significantly faster than purely temperature loops, hinting at a future possibility of time and cost efficient FORC characterization of temperature dependent transitions.
\end{abstract}

Published under license by AIP Publishing. https://doi.org/10.1063/5.0005076

\section{INTRODUCTION}

Magnetic refrigeration and magnetocaloric effect (MCE) are timely topics of research. ${ }^{1}$ Since the discovery of the giant magnetocaloric effect of $\mathrm{Gd}_{5}(\mathrm{Si}, \mathrm{Ge})_{4}$ in $1997,{ }^{2}$ magnetic refrigeration is considered a "promising alternative" for temperature control, producing a surge in the number of publications on the magnetocaloric effect. As these materials enable the possibility of designing magnetic refrigerators with operation temperatures close to room temperature, there has been a noticeable increase in the development of refrigerator prototypes using this principle of operation. ${ }^{1,3}$ This scientific and technological interest is mainly due to the environmental friendliness of magnetic refrigeration, which is energetically more efficient than the conventional refrigerator devices and does not require the use of ozone depleting or greenhouse effect related gases.

While magnetocaloric materials can be classified according to composition, the representation of Ashby plots of the most relevant properties makes the different families of materials merge when composition is slightly tuned. ${ }^{4}$ Therefore, the most efficient criterion to classify magnetocaloric materials is based on the order of their phase transition, which can be either first- (FOPT) or secondorder (SOPT) type. The abrupt change of magnetization vs temperature associated with FOPT materials produces larger magnetic entropy change and adiabatic temperature change than SOPT materials. However, this is accompanied by thermal hysteresis, rate dependent behavior, and decreased cyclic performance, which do not appear in SOPT materials. While the distinction between FOPT and SOPT materials by means of purely magnetic measurements was usually based on indirect or qualitative procedures, there have been recent advances in establishing a quantitative criterion that allows this identification, ${ }^{5}$ which has been shown to be valid for very diverse types of materials such as LaFeSi, Heusler alloys, cobaltites, ${ }^{6}$ and $\mathrm{Tb}_{3} \mathrm{Ni}$ single crystals with peculiar magnetic behavior. ${ }^{7}$ There has also been progress in developing a series of alloys and compounds to reach the intermediate point where the transition changed from FOPT to SOPT, as in $\mathrm{LaFeSi}^{8,9}$ and MnFePSi. ${ }^{10}$

Despite the broad variety of materials reported in the literature, this "promising alternative" has not materialized in the consumer market yet. The reason for this delay in reaching the market has different aspects, but probably the key limitations are related to the performance of the magnetocaloric materials, being thermal hysteresis the most relevant one. However, there are alternative 
applications of magnetocaloric materials, such as thermomagnetic motors, for which there are theoretical studies, indicating that a moderate thermal hysteresis could be beneficial for the performance of the device. ${ }^{11}$ Consequently, as in any other aspect of magnetic materials' research, we should not consider thermal hysteresis as an intrinsically bad characteristic. Let us just recall that for permanent magnet applications, coercivity is a required property, while it has to be avoided in the case of soft magnetic materials; the situation might be analogous with magnetocaloric materials, depending on the target application. In order to advance with the development of magnetocaloric materials toward different possible final uses, we should be able to characterize their thermal hysteresis and, from the knowledge gained, establish strategies to either minimize it when needed (like in magnetic refrigeration applications) or optimize it when it is required (as suggested for thermomagnetic generators).

There have been proposals of new techniques to understand the behavior of FOPT magnetocaloric materials, such as the extension of first order reversal curves (FORCs) ${ }^{12}$ to characterize the interactions between phases in multiphase magnetocaloric materials ${ }^{13}$ and to fingerprint the details of the hysteresis of the thermomagnetic phase transition. ${ }^{14}$ In this latter respect, temperature-FORC (T-FORC) is formally analogous to its more conventional field counterpart, facilitating the study of phase transitions that are driven by temperature. FOPT materials exhibit hysteresis as a function of the driving force that causes the transition (temperature in our case). Minor loops measured from saturation to a certain reversal temperature, $T_{r}$, and back to saturation are recorded for different values of $\mathrm{T}_{\mathrm{r}}$. The set of reversal curves from $T_{r}$ up to saturation is subsequently processed to obtain the distribution of hysterons that globally represent the hysteretic behavior of the sample and allow fingerprinting the details of the hysteretic transition,

$$
\rho\left(T, T_{r}\right)=-\frac{\partial^{2} M\left(T, T_{r}\right)}{\partial T_{r} \partial T} .
$$

In the case of SOPT materials, there is no thermal hysteresis associated with the transition, and, therefore, T-FORC experiments cannot be performed. In this case, more conventional field FORC can be performed to gain insight into the magnetic interactions present among different magnetocaloric phases. ${ }^{1}$

T-FORC has allowed confirming that the details of the transformation in Heusler alloys depend on the two extensive variables, temperature and field. ${ }^{14}$ However, the comparison between the different distributions for different applied fields could only be made qualitatively because the temperature axis has to be arbitrarily shifted for each applied field. In this work, we revisit T-FORC analysis by considering a unified driving force approach that allows us to avoid this drawback of the previous procedure. By using an effective temperature $\left(T^{*}\right)$, the combined effect of field and temperature can be analyzed and compared, leading to $\mathrm{T}^{\star}$ FORC. In addition, a possible method to reduce the experimental time in this timeconsuming set of experiments is proposed.

\section{EXPERIMENTAL}

A Heusler alloy sample with composition $\mathrm{Ni}_{45.7} \mathrm{Mn}_{36.6} \mathrm{In}_{13.5} \mathrm{Co}_{4.2}$ was kindly provided by Dr. Tino Gottschall and Professor Oliver
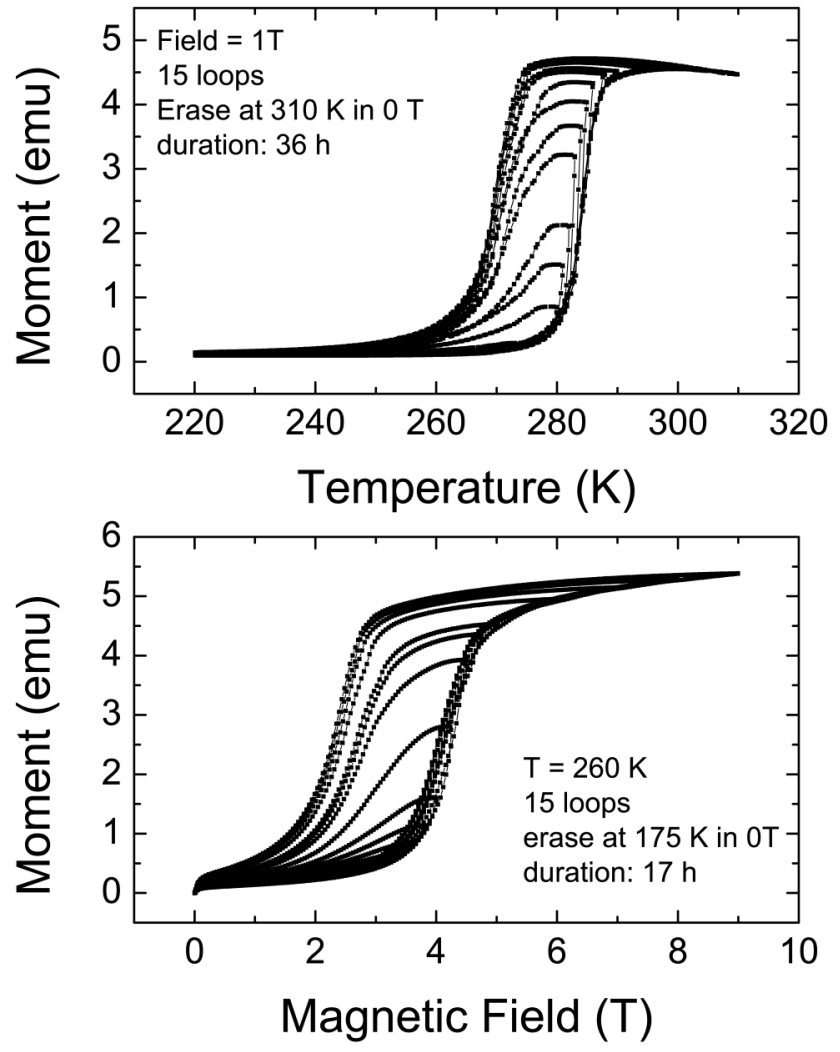

FIG. 1. Temperature and field-dependent magnetization loops, showing that the austenitic transition can be driven both by temperature and magnetic field. In between loops, the sample was taken to a completely saturated state (see Experimental for details of the procedure).

Gutfleisch. Further details of sample preparation as well as compositional and microstructural characterization can be found elsewhere. ${ }^{15}$

The temperature and field-dependent magnetization curves were measured in a vibrating sample magnetometer using a maximum applied field of 9T. The first order reversal curves of magnetization vs temperature upon cooling were recorded for applied fields of $1 \mathrm{~T}$ and 2T. As seen in Fig. 1, the martensitic-austenitic transition can be driven both by temperature and by a magnetic field. Therefore, as FORC analysis requires the saturation of the transformation in between experiments to have the sample in a reproducible state, the sample was heated well above the transition temperature (up to $310 \mathrm{~K}$ ) in zero field in between each minor loop measurement. For field-dependent loops, the sample was cooled down in zero field well below the transition temperature (down to $175 \mathrm{~K}$ ). In both cases, a dwell time of $100 \mathrm{~s}$ at the reset temperature was used. Temperature dependent hysteresis loops were measured at a rate of $2 \mathrm{~K} / \mathrm{min}$, while field-dependent hysteresis used a rate of $45 \mathrm{Oe} / \mathrm{s}$.

\section{RESULTS AND DISCUSSION}

The use of T-FORC has been previously shown to be suitable to fingerprint details of the thermal hysteresis of magnetocaloric 
materials. These experiments are performed at a constant applied field, and the temperature of the sample is varied between saturation, reversal temperature, and back to saturation. However, when the applied field increases, the saturation hysteresis loop of the studied sample moves to lower temperatures. Figure 2 shows the saturation $\mathrm{M}(\mathrm{T})$ hysteresis loops of the $\mathrm{Ni}_{45.7} \mathrm{Mn}_{36.6} \mathrm{In}_{13.5} \mathrm{Co}_{4.2}$ sample for applied fields of $1 \mathrm{~T}$ and $2 \mathrm{~T}$, where the center of the loop (marked as a vertical dashed line) shifts from $277 \mathrm{~K}$ to $270 \mathrm{~K}$ with increasing field.

Consequently, to be able to compare the T-FORC distributions of the same sample under different field conditions, the temperature axis has to be referred to the center of the saturation loop. This has the undesired effect of giving positive and negative $T_{u}$ temperatures in the vertical distribution axis. Therefore, the translation of the origin prevents a comparison of the details of the distribution. However, it is still possible to qualitatively compare their shape. As shown in Fig. 3 (adapted from Ref. 14), both distributions show analogous features, with a major peak $(\mathrm{Pk} 1)$ located at larger $\mathrm{T}_{\mathrm{h}}$ temperatures and a minor peak $(\mathrm{Pk} 2)$ located at smaller $\mathrm{T}_{\mathrm{h}}$. However, it is not possible to investigate in this way if the distribution shifts with the field, as the experimental temperature axis

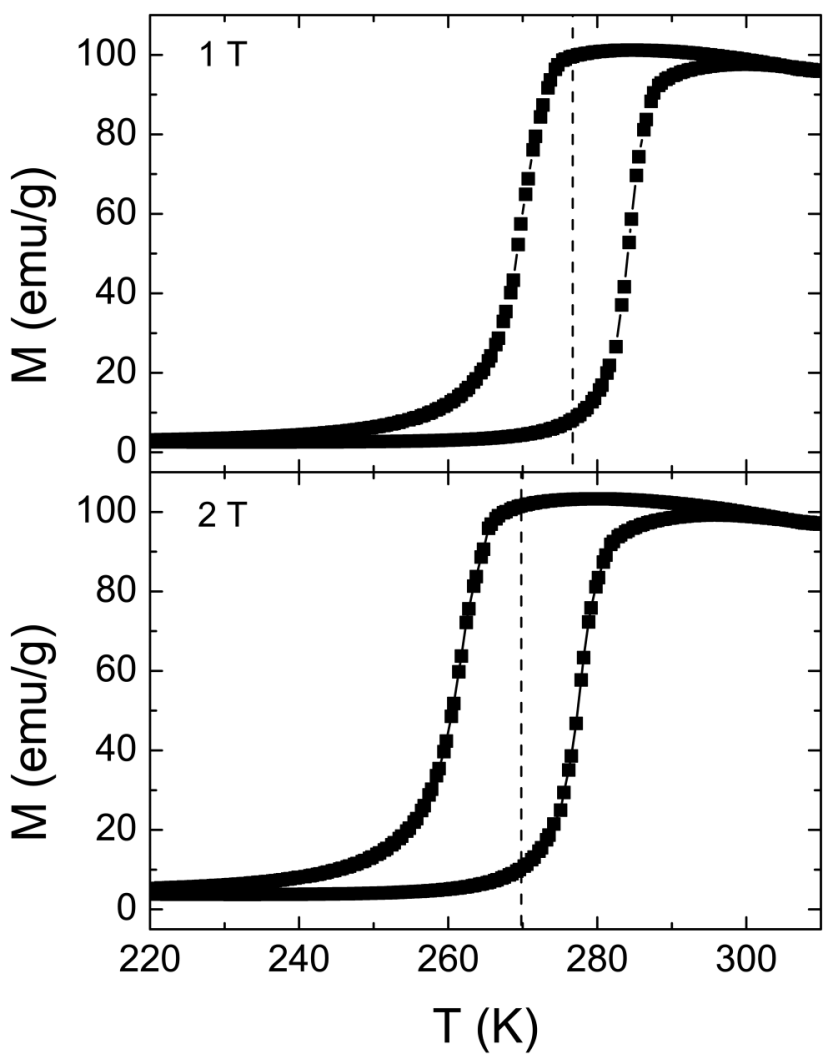

FIG. 2. Thermal hysteresis of $\mathrm{Ni}_{45.7} \mathrm{Mn}_{36.6} \mathrm{In}_{13.5} \mathrm{Co}_{4.2}$ for applied fields of $1 \mathrm{~T}$ and $2 \mathrm{~T}$. The center of the saturation loop, shifting to lower temperatures with increasing field, is marked as vertical dashed lines.

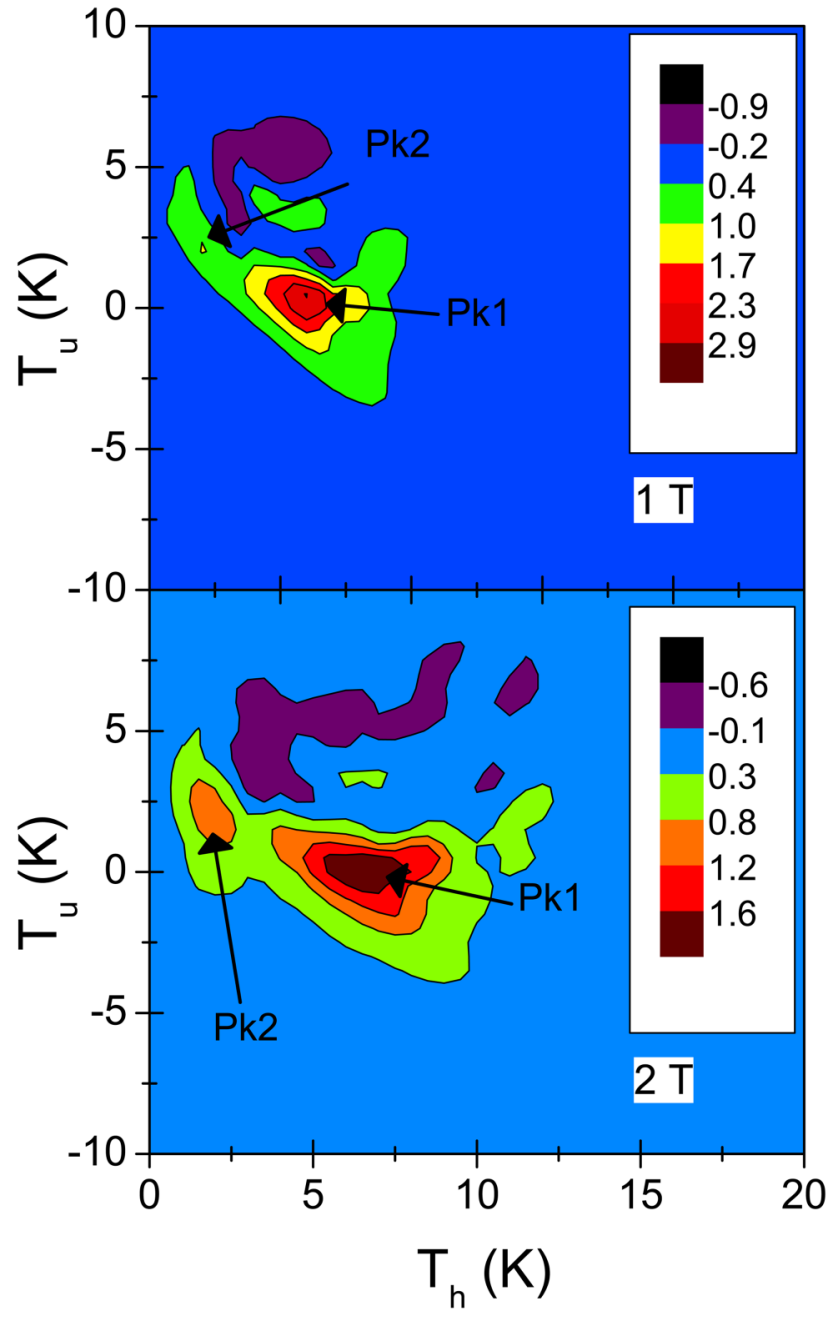

FIG. 3. T-FORC distribution of $\mathrm{Ni}_{45.7} \mathrm{Mn}_{36.6} \mathrm{In}_{13.5} \mathrm{CO}_{4.2}$ for applied fields of $1 \mathrm{~T}$ and 2T. Adapted from Ref. 14.

had to be shifted to the center of the saturation loop before processing the distributions.

To solve this difficulty, we can take into account that the magneto-structural phase transition in Ni-Mn-In-Co can be driven both by temperature and by a magnetic field. Both driving forces provide energy to the sample in order to advance along the phase transformation. This is formally similar to the case of the $\mathrm{M}(\mathrm{H})$ hysteresis loops of interacting superparamagnetic nanoparticles, where both field and temperature have an (competing) effect on the value of magnetization. In that case, a phenomenological approach that linked the effect of temperature and field on the magnetic properties via an effective temperature could be proposed. ${ }^{16}$ Despite the intrinsic differences between the two experimental systems, it was recently demonstrated that, following a similar approach, it is possible to define an effective temperature 
$T^{*}$ that accounts for the energy (either thermal or magnetic) supplied to the magnetocaloric sample in order to drive the transition. ${ }^{17}$ This effective temperature is defined as

$$
T^{*}=T+\frac{N_{A} m_{S}}{M \Delta S_{s t r}} \mu_{0} H
$$

where $\mu_{0}$ is the magnetic permeability of vacuum, $N_{A}$ is the Avogadro constant, $M$ is the molar mass, $m_{S}$ is the saturation magnetic moment per atom, and $\Delta S_{t r}$ is the entropy change of the transition per unit mass. Unlike the case of superparamagnetic nanoparticles, the effective temperature is directly proportional to both temperature and field, as both make the material transform into the other phase.

Analogous to the T-FORC distributions as a function of $T_{\mathrm{h}}$ and $T_{\mathrm{u}}$, the effective temperature approach allows us to express the distributions as a function of effective magnitudes $T^{*}$ and $T^{*}$, related to the original magnitudes via Eq. (2), obtaining $T^{\star}$ FORC representations. These transformed distributions for applied fields of $1 \mathrm{~T}$ and $2 \mathrm{~T}$ are shown in Fig. 4 . We can notice that now the vertical axis does not have to be referred to the center of the saturation loops, but it is expressed as absolute temperature. This allows for a more reliable comparison of the distributions as a function of field. Qualitatively, features are the same as with the untransformed variable, with distributions consisting of a major $(\mathrm{Pk} 1)$ and a minor $(\mathrm{Pk} 2)$ peak. It can be observed that while the location of the major and minor peaks of the distribution $(\mathrm{Pk} 1$ and $\mathrm{Pk} 2)$ does not displace along the vertical axis with the application of a magnetic field, there is a minor influence of the magnetic field on the location of the major peak along the horizontal axis, suggesting the eventual appearance of a kinetic arrest, which was experimentally found for this sample. ${ }^{1,3}$

In addition to a more quantitative comparison of the features of the distributions, the use of the unified driving force, combining thermal and magnetic energies, would allow performing $\mathrm{T}^{*} \mathrm{FORC}$ experiments in a shorter experimental time than the one required for T-FORC. One of the major limitations of T-FORC is the long time required to collect the experimental data. Figure 1 shows, for comparison, the typical set of experiments needed for a FORC distribution as a function of temperature or as a function of field. While thermal experiments took $36 \mathrm{~h}$ to be performed, including the time for the actual measurements plus the time for erasing the memory of the sample in between experiments, field loops would only take $17 \mathrm{~h}$, allowing for a more effective use of experimental time when minor loops have to be collected. The use of $T^{\star}$ will allow us to compare the information of different field and temperature loops. However, to be able to make an accurate comparison between the field and temperature-FORC distributions in the effective temperature representation, it would be necessary to use a material with a larger separation between the martensitic transition and the Curie temperature of the austenite. It would also be advantageous to have a more gradual transition in the temperature scale, to be able to capture a finer resolution in the reversal temperature axis of the FORC distribution (currently, the finer resolution in $\mathrm{T}_{\mathrm{r}}$ of Fig. 1 is limited to $1 \mathrm{~K}$ due to instrumental constraints).

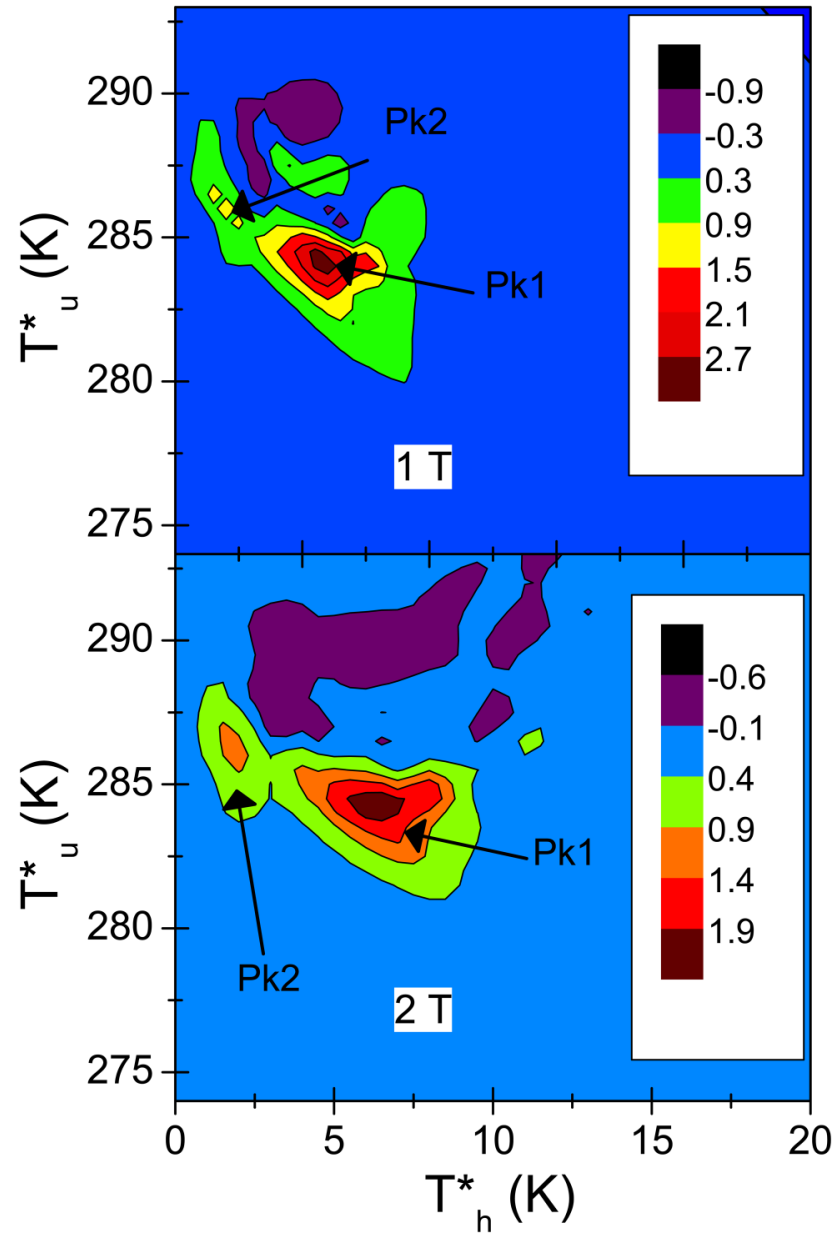

FIG. 4. $T^{*} F O R C$ distributions of $\mathrm{Ni}_{45.7} \mathrm{Mn}_{36.6} \mid \mathrm{In}_{13.5} \mathrm{CO}_{4.2}$ for applied fields of $1 \mathrm{~T}$ and $2 \mathrm{~T}$.

This approach can also be extended to other types of phase transformations, for which the magnetic field is replaced by other driving forces (such as the electric field, pressure, or combinations of several of them). In addition, it has to be taken into account that any hysteretic process would be rate dependent. This is particularly true for the type of magnetocaloric materials considered in this study. There have been previous studies in the literature analyzing the influence of rate dependent hysteresis in FORC. ${ }^{18,19}$ Those authors use a phenomenological model suitable to describe the influence of the speed of the application of the driving force to be able to model the effects on the FORC distribution. In the case of magnetocaloric materials, a general kinetic model of the transformation should be developed, but that is beyond the scope of the current work. Therefore, at the current stage, this $\mathrm{T}^{*} \mathrm{FORC}$ approach can be used for fixed excitation frequencies (typically similar to those used in experimental refrigeration devices), with the possibility extending it to a frequency-independent approach 
once the general kinetic model of the phase transformation is developed.

\section{CONCLUSIONS}

The use of an effective temperature that combines the effect of the different driving forces of the austenitic transition in $\mathrm{Ni}_{45.7} \mathrm{Mn}_{36.6} \mathrm{In}_{13.5} \mathrm{Co}_{4.2}$ allows for a more quantitative comparison of the FORC distributions obtained under different experimental conditions. The shift of the distribution toward larger effective hysteretic temperatures indicates the existence of a kinetic arrest of the transformation when the field is increased.

The use of this unified driving force approach also allows for a faster measurement of the reversal curves required for the $\mathrm{T}^{\star} \mathrm{FORC}$ distributions, being a promising method for a more efficient characterization of temperature and field-dependent hysteretic behavior, as well as for transformations affected by other driving forces.

\section{ACKNOWLEDGMENTS}

This work was supported by AEI/FEDER-UE (Project No. MAT-2016-77265-R), FEDER Operating Program 2014-2020 and Consejería de Economía y Conocimiento of the Regional Government of Andalucia (No. US-1260179), Plan Andaluz de Investigación, Desarrollo e Innovación (No. P18-RT-746), and Army Research Laboratory under Cooperative Agreement No. W911NF-19-2-0212. Samples were kindly provided by Dr. Tino Gottschall and Professor Oliver Gutfleisch. The data that support the findings of this study are available from the corresponding author upon reasonable request.

\section{REFERENCES}

${ }^{1}$ V. Franco, J. S. Blázquez, J. J. Ipus, J. Y. Law, L. M. Moreno-Ramírez, and A. Conde, Prog. Mater. Sci. 93, 112 (2018).

${ }^{2}$ V. K. Pecharsky and K. A. Gschneidner, Phys. Rev. Lett. 78, 4494 (1997).

${ }^{3}$ B. Yu, M. Liu, P. W. Egolf, and A. Kitanovski, Int. J. Refrig. 33, 1029 (2010).

${ }^{4}$ V. Franco, J. S. Blázquez, B. Ingale, and A. Conde, Annu. Rev. Mater. Res. 42, 305 (2012).

${ }^{5}$ J. Y. Law, V. Franco, L. M. Moreno-Ramirez, A. Conde, D. Y. Karpenkov, I. Radulov, K. P. Skokov, and O. Gutfleisch, Nat. Commun. 9, 2680 (2018).

${ }^{6}$ J. Y. Law, V. Franco, A. Conde, S. J. Skinner, and S. S. Pramana, J. Alloys Compd. 777, 1080 (2019)

${ }^{7}$ A. Herrero, A. Oleaga, A. F. Gubkin, A. Salazar, and N. V. Baranov, J. Alloys Compd. 808, 151720 (2019).

${ }^{8}$ O. Gutfleisch, A. Yan, and K.-H. Muller, J. Appl. Phys. 97, 10 M305 (2005).

${ }^{9}$ J. Liu, J. D. Moore, K. P. Skokov, M. Krautz, K. Lowe, A. Barcza, M. Katter, and O. Gutfleisch, Scr. Mater. 67, 584 (2012).

${ }^{10}$ M. F. J. Boeije, M. Maschek, X. F. Miao, N. V. Thang, N. H. van Dijk, and E. Bruck, J. Phys. D: Appl. Phys. 50, 174002 (2017).

${ }^{11}$ C. V. X. Bessa, L. D. R. Ferreira, O. Horikawa, J. C. B. Monteiro, F. G. Gandra, and S. Gama, J. Appl. Phys. 122, 244502 (2017).

${ }^{12}$ I. Mayergoyz, IEEE Trans. Magn. 22, 603 (1986)

${ }^{13}$ V. Franco, F. Béron, K. R. Pirota, M. Knobel, and M. A. Willard, J. Appl. Phys. 117, 17C124 (2015).

${ }^{14}$ V. Franco, T. Gottschall, K. P. Skokov, and O. Gutfleisch, IEEE Magn. Lett. 7, 1 (2016).

${ }^{15}$ T. Gottschall, K. P. Skokov, B. Frincu, and O. Gutfleisch, Appl. Phys. Lett. 106, 021901 (2015)

${ }^{16}$ V. Franco, C. F. Conde, A. Conde, and L. F. Kiss, Phys. Rev. B 72, 174424 (2005).

${ }^{17}$ J. S. Blázquez, V. Franco, A. Conde, T. Gottschall, K. P. Skokov, and O. Gutfleisch, Appl. Phys. Lett. 109, 122410 (2016).

${ }^{18}$ C. Enachescu, R. Tanasa, A. Stancu, G. Chastanet, J.-F. Létard, J. Linares, and F. Varret, J. Appl. Phys. 99, 08J504 (2006).

${ }^{19}$ D. Cimpoesu, I. Dumitru, and A. Stancu, J. Appl. Phys. 120, 173902 (2016). 